\title{
Study of single top production at high energy electron positron colliders
}

\author{
J. Fuster, I. García, P. Gomis, M. Perelló, E. Ros, M. Vos ${ }^{\mathrm{a}}$ \\ IFIC, University of Valencia-CSIC, Valencia, Spain
}

Received: 14 January 2015 / Accepted: 11 May 2015 / Published online: 22 May 2015

(C) The Author(s) 2015. This article is published with open access at Springerlink.com

\begin{abstract}
The effect of single top production on the study of top quark pair production in future high energy electronpositron colliders is evaluated. The rate of the single top quark production process is sizeable throughout a large range of center-of-mass energies and the final state cannot easily be distinguished from the dominant pair production process. We discuss the impact on the top quark mass extraction from a scan through the pair production threshold and the determination of top quark form factors in the continuum. These results advocate for the exploration of the inclusive $e^{+} e^{-} \rightarrow W^{+} b W^{-} \bar{b}$ process, that includes both top quark pair and single top quark production.
\end{abstract}

\section{Introduction}

A high-luminosity, high-energy, linear $e^{+} e^{-}$collider yields excellent opportunities for precision tests of the Standard Model of particle physics. The combination of precisely calculable electroweak production and strict control of the initial state with the relatively benign experimental environment and state-of-the-art detector systems allow for a characterization of Standard Model and new physics processes with a precision that goes well beyond what can be achieved at hadron colliders.

Two projects of linear electron-positron colliders are being considered: the International Linear Collider (ILC $[1,2]$ ) and the Compact Linear Collider (CLIC [3]). The physics case for a linear $e^{+} e^{-}$machine has been made in great detail in Refs. [4-11]. The specific case of a multi-TeV $e^{+} e^{-}$collider is discussed in Refs. [12-14]. In both cases, the center-of-mass energy will exceed $\sqrt{s}=$ $350 \mathrm{GeV}$, the threshold for top quark pair production. Unlike other quarks, the top quark has never been produced in $e^{+} e^{-}$machines, and therefore a precise measurement of electroweak top quark pair production is missing. The study of top quark properties is therefore one

\footnotetext{
a e-mail: marcel.vos@ific.uv.es
}

of the most exciting prospects for a future linear collider [15]. Detailed full-simulation studies have been made of the prospects for a precise top quark mass measurement $[16,17]$ and characterization of the $t \bar{t} Z$ and $t \bar{t} \gamma$ vertices [18].

Single top production, through the $e^{+} e^{-} \rightarrow W^{-} t \bar{b}, W^{+} \bar{t} b$ process depicted in the central panel of Fig. 1, is abundant at $e^{+} e^{-}$colliders that operate at $\sqrt{s}>300 \mathrm{GeV}$. Note that for the $t \rightarrow b W$ decay, this process gives rise to the same $W^{+} b W^{-} \bar{b}$ final state as top pair production. Using MADGRAPH we find ten leading-order diagrams of this type, against two for top quark production. A third group of processes gives rise to the same final state: $W W Z$, $W W h$ and $W W \gamma$ production, with $Z / \gamma / h \rightarrow b \bar{b}$. At treelevel there are approximately 50 diagrams that produce two $W$-bosons and two $b$-quarks that do not involve top quarks.

Ultimately, all three processes yield the same set of six-fermion final states. In many studies of the linear collider prospects for top physics, single top quark production has been neglected. Notable exceptions are found in Refs. [19,20].

A fully consistent analysis of the inclusive $e^{+} e^{-} \rightarrow$ $W^{+} b W^{-} \bar{b}$ is currently impossible. Even if event generation for the ILC TDR routinely included the full $2 \rightarrow 6$ matrix element at leading order, higher-order corrections for this process are not available for the extraction of top quark properties and couplings. In the continuum, results for the cross-section of top quark pair production are available at NNLO. Cross-sections for the process $W^{+} b W^{-} \bar{b}$ are for the moment only available at $\mathrm{LO}$, but are required at least at NLO (note that NLO calculations are already available for the LHC).

In this note we investigate the impact of single top events in the study of top quark pair production and propose a more inclusive experimental strategy that compares precise predictions for the $e^{+} e^{-} \rightarrow W^{+} b W^{-} \bar{b}$ process to measurements. 


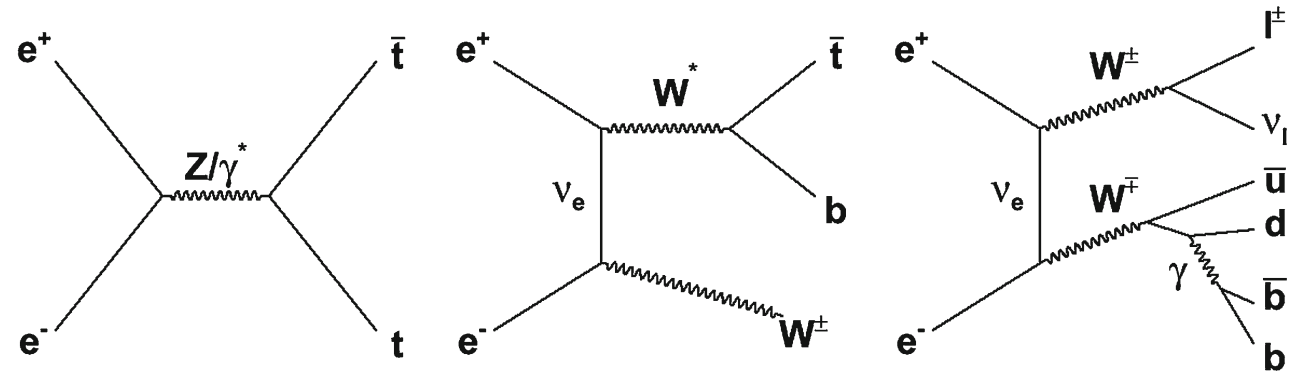

Fig. 1 Feynman diagrams for top quark pair production at a linear collider $\left(e^{+} e \rightarrow Z / \gamma^{*} \rightarrow t \bar{t}\right.$, left panel), single top production $\left(e^{+} e^{-} \rightarrow\right.$ $W^{-} t \bar{b}, W^{+} \bar{t} b$, central panel $)$, and triple gauge boson production $\left(e^{+} e^{-} \rightarrow W^{+} W^{-} Z\right.$, right panel $)$

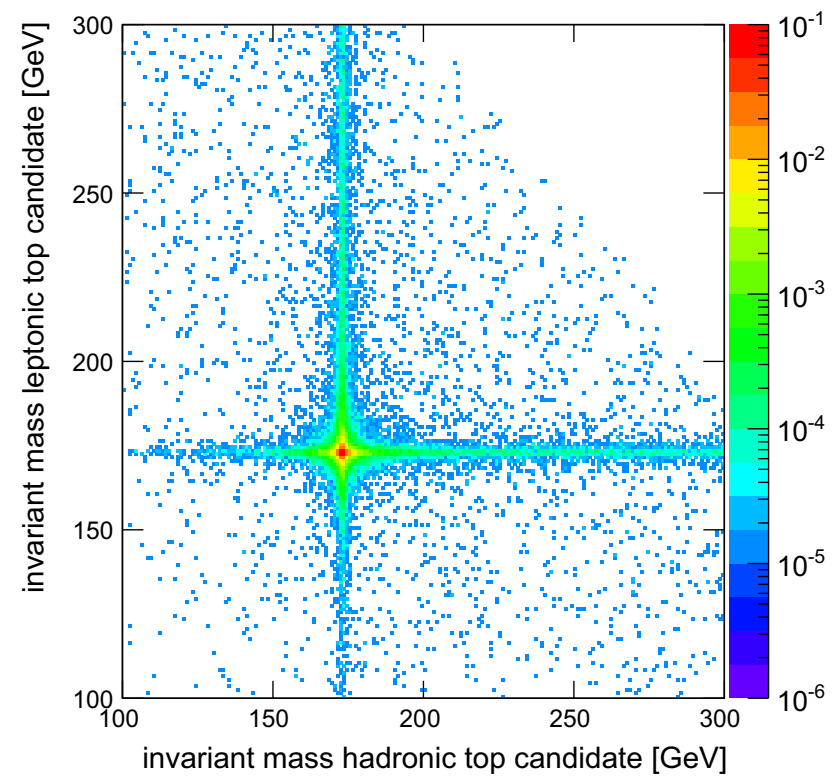

Fig. 2 Reconstructed invariant mass at thruth level of the hadronic versus the leptonic side of $t \bar{t} \rightarrow W^{+} b W^{-} \bar{b}$ events. The events cluster around the input top mass value, but one can observe a significant amount of off-shell events

\section{Distinguishing single top from top quark pair production}

As top quark pair production and single top quark production give rise to the same six-fermion final state, the question arises as to how one can distinguish both sources. At a fundamental level the single top and top quark pair production processes are entangled by interference between the different diagrams. No algorithm can ever separate them fully. However, one could hope to use some of the marked features of the $e^{+} e^{-} \rightarrow t \bar{t}$ process to make it stand out among the other processes that give rise to the $W^{+} b W^{-} \bar{b}$ final state. One could then hope to isolate samples that are enriched in top quark pairs or single top quark events.

In Fig. 2 we present the invariant mass of the $W^{+} b$ and $W^{-} \bar{b}$ combinations at truth level using $e^{+} e^{-} \rightarrow t \bar{t} \rightarrow$

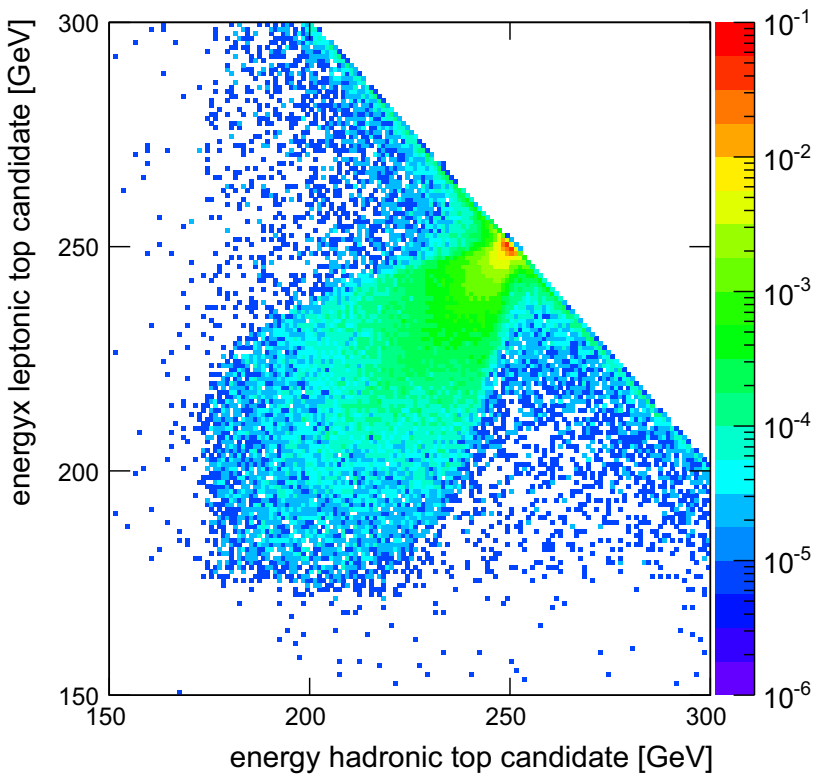

Fig. 3 Reconstructed beam energy at thruth level of the hadronic versus the leptonic side of $W^{+} b W \bar{b}$ events. The center-of-mass energy is $500 \mathrm{GeV}$, so double-top events cluster at beam energies of $250 \mathrm{GeV}$. In single-top events, the beam energy follows the diagonal $E_{l e p}+E_{\text {had }}=$ $500 \mathrm{GeV}$

$W^{+} b W^{-} \bar{b}$ events generated with WHIZARD [21,22] at $\sqrt{s}=500 \mathrm{GeV}$, including the effect of initial state radiation and the beam energy spread expected at the ILC. The majority of events is found around $m_{W^{+} b} \sim m_{W^{-} \bar{b}} \sim m_{t}$. However, in a significant fraction of events one of the $W$ boson $b$-quark pairs has an invariant far from the top quark mass. The cross is populated by a mixture of $t \bar{t}$ events with an off-shell top quark and genuine single-top production through diagrams such as that in the central panel of Fig. 1

In Fig. 3 we present the reconstructed beam energy of the $W b$ decaying leptonically versus the $W b$ decaying hadronically. The energy of the $W b$ pair tends to cluster at about half the center-of-mass energy, as expected for double-top events. We observe however a significant fraction of events along the diagonal $E_{l e p}+E_{\text {had }}=\sqrt{s}$. Those are mostly single top events. This figure suggests another potential cri- 
terium for partial separation of single and double-top events using the reconstructed beam energy.

These figures suggest an (ad-hoc) truth-level categorisation of events according to the number of on-shell top quark candidates, that is used to quantify the non-t $\bar{t}$ contribution to $e^{+} e^{-} \rightarrow W^{+} b W^{-} \bar{b}$ production in the remainder of this paper. We consider the event to correspond to top quark pair production whenever the following relation is satisfied for both $W b$ combinations:

$$
\left|m_{W b}-m_{t}^{M C}\right|<15 \mathrm{GeV}
$$

Events that meet this criterium only for one of the $W b$ pairs are labeled as single top quark events, and the remaining events are considered as non-top events.

With this criterion we find that at $500 \mathrm{GeV}$ typically $90 \%$ of $e^{+} e^{-} \rightarrow W^{+} b W^{-} \bar{b}$ events is formed by top quark pairs, $9 \%$ corresponds to single top production and only $1 \%$ stems from processes that do not involve top quarks. These fractions depend strongly on the center-of-mass energy of the collider (more in Sect. 5) and on the beam polarization (more in Sect. 3). Over a broad range of energies and operating scenarios the fraction of single-tops is far from negligible and may have a significant impact on the measurement of top quark properties and the search for signs of new physics in $t \bar{t}$ production at lepton colliders.

\section{Experimental study at $\sqrt{s}=500 \mathrm{GeV}$}

In this section we concentrate on the impact of realistic analysis cuts on the composition of the $e^{+} e^{-} \rightarrow W^{+} b W^{-} \bar{b}$ sample at $\sqrt{s}=500 \mathrm{GeV}$. The analysis is based on the study of cross-sections and asymmetries at $\sqrt{s}=500 \mathrm{GeV}$ in Ref. [18]. Final states of the type $l v q_{1} q_{2} b_{1} b_{2}$ are generated using the WHIZARD Monte Carlo program. These events are mostly $W^{+} b W^{-} \bar{b}$ events, where one $W$ decays hadronically and the other leptonically. Some 100,000 MC events are processed in a detailed simulation of the ILD detector [23,24] response based on GEANT4 [25]. Events are reconstructed, to the level of particle flow objects and jets, using the ILC software package. In these samples the beams are $100 \%$ polarized.
First, we discuss briefly the single top content as a function of beam polarization. The fraction of $W^{+} b W^{-} \bar{b}$ events that corresponds to single top quark production is quite sensitive to the polarization of the electron and positron beams. We evaluate the fractions of top quark pair, single top, and non-top events using the criterion of Eq. (2.1) on $e^{+} e^{-} \rightarrow e l v q_{1} q_{2} b_{1} b_{2}$ samples generated with WHIZARD, including initial state radiation and a realistic ILC luminosity profile. For a fully left-handed electron beam and fully right-handed positron beam $\left(e_{L}^{-} e_{R}^{+}\right)$the total cross-section is nearly three times larger. The top quark pair fraction remains $90.2 \%$. The single top and non-top fractions are $8.9 \%$ and $0.9 \%$, respectively. For the opposite $\left(e_{R}^{-} e_{L}^{+}\right)$configuration, the total cross-section is similar to the unpolarized result. The top quark pairs make up $94.3 \%$ of the sample, with only $5.6 \%$ of single top quarks and about $0.1 \%$ for non-top production.

The selection in Ref. [18] rejects background and improves the fraction of well-reconstructed events using a cut on a $\chi^{2}$ formed by the reconstructed top mass, beam energy and bquark energy in the top rest frame. Only the hadronically decaying top is used in the analysis. Table 1 summarizes the selection efficiencies at various stages of the analysis.

The preselection includes the lepton reconstruction and identification, and the requirement of two b-tagged jets. The results in the second row are obtained after the cut on the hadronic top $\chi^{2}<15$ employed in Ref. [18]. We observe that for both beam polarizations the efficiency for single top events is significantly smaller than for top quark pairs. This is expected, since the $W b$ pair mass and the beam energy are required to be compatible with the values expected for top quark pair production in the $\chi^{2}$ cut.

The inclusion of cuts on the leptonic top in the $\chi^{2}$ does not improve the rejection. The third row in Table 1 corresponds to cuts on both the hadronic and leptonic top $\chi^{2}$, where the $\chi^{2}$ cut is adjusted to obtain the same efficiency on the total sample. This can be understood as follows: once an event passes the beam energy constraint, momentum conservation (forced by the neutrino reconstruction) imposes a similar invariant mass on both the hadronic and leptonic sides of the event, whether the event is a single or a double top. In other words, the leptonic side does not provide any additional background rejection.
Table 1 Selection efficiencies for various events samples. The efficiency estimates are based on 140,000 events for $e_{L}^{-} e_{R}^{+}$and $40,000 e_{R}^{-} e_{L}^{+}$event and thus have a statistical error of several per mil

\begin{tabular}{|c|c|c|c|c|c|c|}
\hline \multirow[t]{2}{*}{ All } & \multicolumn{3}{|l|}{$e_{L}^{-} e_{R}^{+}$} & \multicolumn{3}{|l|}{$e_{R}^{-} e_{L}^{+}$} \\
\hline & $t \bar{t}(\%)$ & Single top $(\%)$ & $W^{+} b W^{-} \bar{b}(\%)$ & $t \bar{t}(\%)$ & Single top $(\%)$ & $W^{+} b W^{-} \bar{b}(\%)$ \\
\hline Preselection & 48 & 45 & 47 & 49 & 48 & 49 \\
\hline $\begin{array}{l}\chi^{2} \text { on hadronic top } \\
\text { candidate }\end{array}$ & 33 & 20 & 31 & 40 & 31 & 40 \\
\hline $\begin{array}{l}\chi^{2} \text { on both top } \\
\text { candidates }\end{array}$ & 34 & 22 & 32 & 30 & 20 & 29 \\
\hline
\end{tabular}


Table 2 Non- $t \bar{t}$ and single top content of the $W^{+} b W^{-} \bar{b}$ sample, as a function of the center-of-mass energy. The first two columns correspond to a leading-order calculation for unpolarized and polarized beams. Fur- ther results correspond to the $W^{+} b W^{-} \bar{b}$ including QCD bound state effects. In the last two columns initial-state-radiation and the ILC beam energy spread are taken into account

\begin{tabular}{|c|c|c|c|c|c|c|}
\hline \multirow[t]{2}{*}{$\sqrt{s}(\mathrm{GeV})$} & \multirow{2}{*}{$\begin{array}{l}e^{-} e^{+}(\mathrm{LO}) \\
\text { Single top }(\%)\end{array}$} & \multirow{2}{*}{$\begin{array}{l}e_{L}^{-} e_{R}^{+}(\mathrm{LO}) \\
\text { Single top }(\%)\end{array}$} & \multicolumn{2}{|l|}{$e^{-} e^{+}(\mathrm{NLO})$} & \multicolumn{2}{|c|}{$e^{-} e^{+}(\mathrm{NLO}, \mathrm{ISR}, \mathrm{LS})$} \\
\hline & & & Non- $t \bar{t}(\%)$ & Single top (\%) & Non- $t \bar{t}(\%)$ & Single top $(\%)$ \\
\hline 344 & 23 & 36 & 32 & 24 & 38.2 & 31.1 \\
\hline 345 & 19 & 30 & 22 & 16 & 27.5 & 22.3 \\
\hline 346 & 13 & 26 & 15 & 10 & 20.1 & 16.3 \\
\hline 347 & 9 & 19 & 9 & 5.4 & 12.4 & 9.8 \\
\hline 348 & 7 & 14 & 6 & 4.0 & 7.5 & 5.9 \\
\hline 349 & 5 & 10 & 5 & 3.4 & 5.5 & 4.4 \\
\hline
\end{tabular}

The selection described here is insufficient to reduce the single top contamination to a safe level. The fraction of single top events continues to be large ( $5 \%$ in the present example). At this level it is expected to have a non-negligible effect on the cross-section measurement. An attempt to describe the remaining contamination using Monte Carlo events is likely to bring sizeable systematic uncertainties.

The forward-backward asymmetry is even more sensitive, since the asymmetry of single top production is very small compared to that of top quark pair production. For the $e_{L}^{-} e_{R}^{+}$ beam polarization the asymmetry of the $W b$ system in inclusive $W^{+} b W^{-} \bar{b}$ production process is $6 \%$ smaller than the forward-backward asymmetry in top quark pair production, a relative difference of $14 \%$.

We conclude, therefore, that cuts on the properties of the reconstructed top candidates are unlikely to reduce single top quark production to the level where it has a negligible impact on observables such as the cross-section and forwardbackward asymmetry.

\section{Analysis of top mass at threshold}

As pointed out a long time ago, the cross-section of the $e^{+} e^{-} \rightarrow t \bar{t}$ at threshold could be used to measure in a precise and well defined way the top mass [26]. Detailed calculations are present in Ref. [27] and a complete study of the extraction of top quark properties was performed in Ref. [28].

Recent reanalyses include realistic beam energy spectra for the ILC and CLIC [16] and beam polarization [17]. In the following we use the former analysis as a reference. The selection of events includes a kinematic fit, but as we showed in the previous section, the leptonic side of the event brings no additional rejection of single top events, since the neutrino can always be adjusted to fake a second top. This analysis is very detailed, but single top events have not been included. This paper includes for the case of ILC the program TOPPIK, a NNLO calculation of the cross-section including the $1 \mathrm{~S}$ resonance, initial state radiation and the ILC luminosity

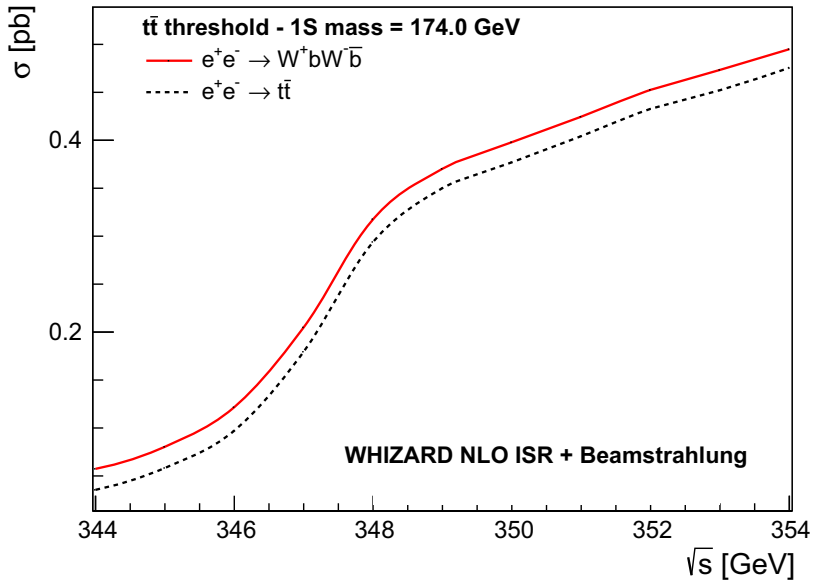

Fig. 4 Cross-sections at NLO, according to WHIZARD, for $W^{+} b W^{-} \bar{b}$ and $t \bar{t}$ production in $e^{+} e^{-}$annihilations with unpolarized beams, as a function of center-of-mass energy, around the double-top threshold region. The 1S-top mass is set to $174 \mathrm{GeV}$

spectrum without beam polarization. For a mass input value of $174 \mathrm{GeV}$ and luminosity of $10 \mathrm{fb}^{-1}$ per data point, a fit is performed in the range $344-354 \mathrm{GeV}$ of center of mass energies. The result is an impressive $27 \mathrm{MeV}$ statistical error for the top mass, and systematic errors well below $100 \mathrm{MeV}$ (a recent paper [29] determines the 4-loop correction for the conversion from $1 S$ to the $\bar{M} S$ scheme; the related theory systematic could be as small as $10 \mathrm{MeV}$, thus prompting a thorough evaluation of the experimental systematic uncertainties).

NLO calculations for the $W^{+} b W^{-} \bar{b}$ process have recently been implemented in WHIZARD around the double-top production threshold including QCD bound state effects. ${ }^{1}$ We use these to estimate the single top fraction in $W b W b$ production. In Table 2 and Fig. 4 the fraction of single top events is given for several center-of-mass energies around the pair production thresholds. The results include the effect of initial state radiation, of a realistic beam energy spectrum for

\footnotetext{
${ }^{1}$ J. Reuter and F. Bach, private communication.
} 
the ILC at this energy, and the effect of polarized beams. The energy range and top quark mass follow the choices in Ref. [16].

We note that the presence of single top events modifies significantly the cross-section. Moreover, the fraction of single top events varies rapidly across the threshold region. This does not mean, however, that the top mass measurement is affected. According to the method proposed in [16], a fit is performed to the cross-section measurement, using a predefined function obtained from the double-top calculation at NNLO. As long as the shape of the curve is not modified, the result is not affected. The $W^{+} b W^{-} \bar{b}$ and $t \bar{t}$ curves are displayed in Fig. 4. We observe that according to the NLO calculation of the $W^{+} b W^{-} \bar{b}$ cross-section, the $t \bar{t}$ crosssection may be obtained quite exactly by shifting down the $W^{+} b W^{-} \bar{b}$ cross-section by some $0.022 \mathrm{pb}$. After fixing this offset with the measurement at $344 \mathrm{GeV}$ we fit the $W^{+} b W^{-} \bar{b}$ shape with the curve for $t \bar{t}$ production. We find the best-fit mass is shifted by $30 \mathrm{MeV}$ with respect to the input mass. This bias is of a similar size as the statistical uncertainty. We emphasize that this estimate should be considered as an upper limit. A more sophisticated treatment is likely to reduce the uncertainty due to the modelling of the single top contamination further. Ultimately, this uncertainty is absent in a fit of the prediction for the inclusive $e^{+} e^{-} \rightarrow W^{+} b W^{-} \bar{b}$ process to the data.

This result is also supported by the NNLL result in Ref. [30], that we reproduce in Fig. 5. The calculations are not directly comparable, as initial state radiation and beam energy spread are not included in Ref. [30]. The authors find that the impact of a cut on the $W b$ mass is an approximately constant shift. The cross-section for $t \bar{t}$ events with $W b$ massin

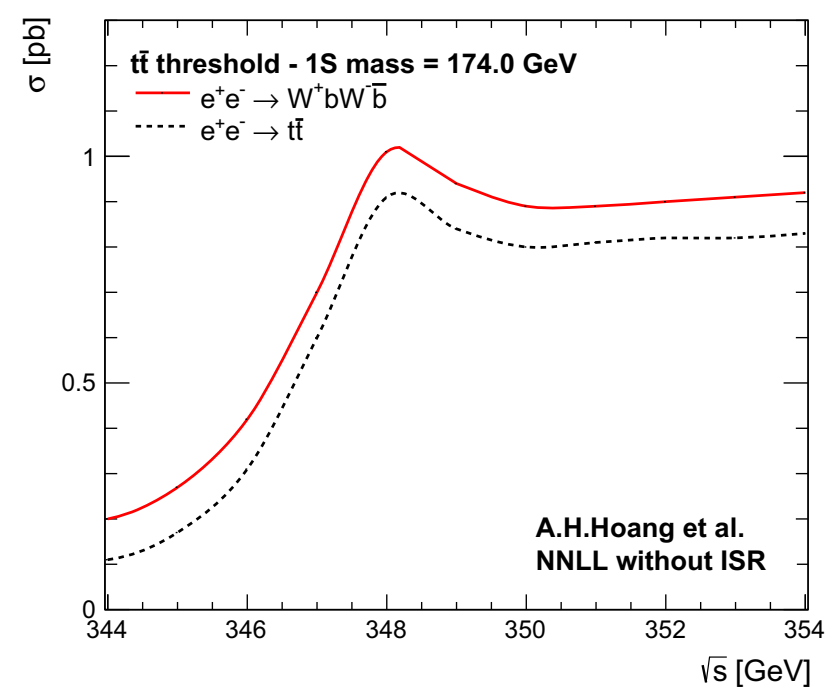

Fig. 5 Cross-sections at NNLL, according to Hoang et al. [30], for $t \bar{t}$ production in $e^{+} e^{-}$annihilations with unpolarized beams a function of center-of-mass energy. The $1 \mathrm{~S}$-top mass is set to $174 \mathrm{GeV}$

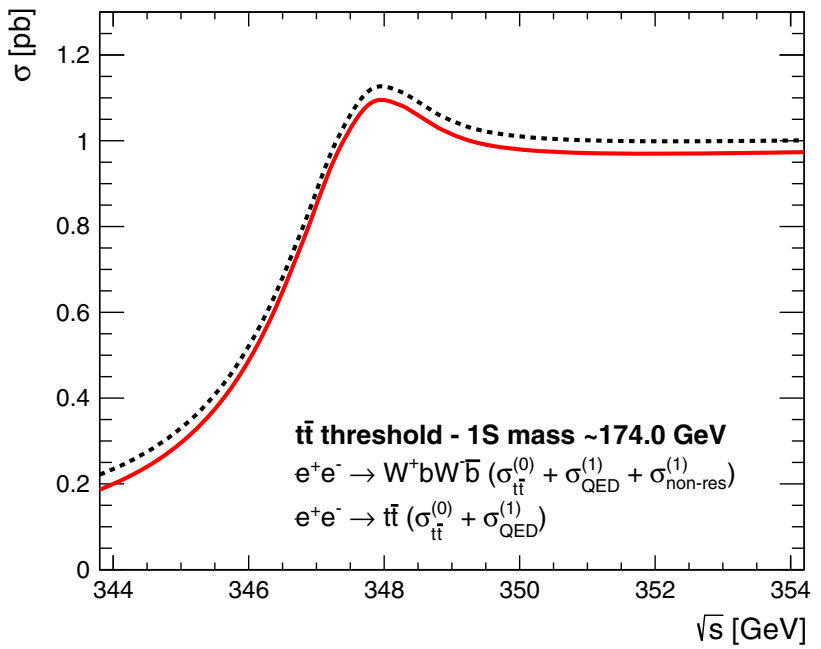

Fig. 6 The effect on the cross-section of non-resonant corrections, according to Beneke et al. [31], for $e^{+} e^{-}$annihilation to $W^{+} b W^{-} \bar{b}$ in the $t \bar{t}$ threshold region. The cross-section for unpolarized beams is given as a function of center-of-mass energy. With respect to the original publication, the curve is shifted such that the $1 \mathrm{~S}$ peak coincides approximately with those of Figs. 4 and 5

a range $-15<m_{W b}-m_{t}<15$ is, as before, shifted down from the inclusive cross-section by 0.050 pb. Finally, Fig. 6 shows the effect of non-resonant effects on the $W b W b$ crosssection. Again, the shift in the cross section is essentially constant across the threshold region.

The single top content of the samples depends strongly on the energy, as seen in Table 2. In analyses where the top quark is reconstructed and cuts are applied on the mass and/or energy of the candidates, the selection efficiency can be quite different for top quark pair and single top events (up to $50 \%$ smaller for single top in the example above). This means that, after correcting for efficiency, the shape of the curve will be deformed if single top events are ignored. Note that this does not apply to Ref. [16], since in this analysis no selection cuts based on reconstructed top quark mass and energy are applied.

As the most precise calculations are only available for the $e^{+} e^{-} \rightarrow t \bar{t}$ process, none of the studies of future collider prospects so far has taken into account the full $W^{+} b W^{-} \bar{b}$ process. As soon as the necessary tools have been developed we suggest to repeat the study of the top quark mass extraction of future lepton colliders using $W^{+} b W^{-} \bar{b}$ events and polarized beams.

\section{Energy dependence}

The composition of the $W^{+} b W^{-} \bar{b}$ sample is energy dependent as shown in Fig. 7, that presents the unpolarized LO cross-sections for $e^{+} e^{-} \rightarrow W^{+} b W^{-} \bar{b}$. The solid line corresponds to the full $2 \rightarrow 4$ process, including single top 


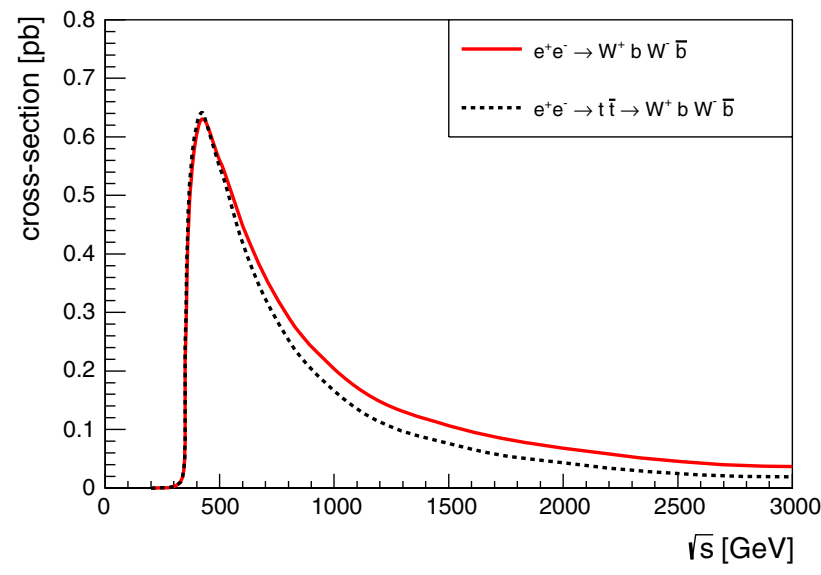

Fig. 7 Cross-section for $W^{+} b W^{-} \bar{b}$ and $t \bar{t}$ events produced in unpolarized electron-positron annihilations as a function of the center-of-mass energy. The curves are obtained using WHIZARD at LO

production and non-top production. The dashed line presents the results for the $e^{+} e^{-} \rightarrow t \bar{t} \rightarrow W^{+} b W^{-} \bar{b}$ process. All results have been obtained using WHIZARD. The difference between the $t \bar{t}$ cross-section and the full $2 \rightarrow 4$ result is $10 \%$ at $500 \mathrm{GeV}$ and rises to $20 \%$ at $1 \mathrm{TeV}$ and nearly $50 \%$ at $3 \mathrm{TeV}$.

The relatively small contribution of the $e^{+} e^{-} \rightarrow t \bar{t}$ process at large center-of-mass energy is confirmed by an analysis of the number of on-shell top quarks $\left(\left|m_{W b}-m_{t}^{M C}\right|<\right.$ $15 \mathrm{GeV}$ ) in $W^{+} b W^{-} \bar{b}$ events at $3 \mathrm{TeV}$. The fraction of events with two on-shell top quarks is $48 \%$, events with a single on-shell top quark make up $35 \%$ of the sample and the remaining $17 \%$ of events has no on-shell top quarks. We conclude that while the rate for the $e^{+} e^{-} \rightarrow t \bar{t}$ process drops at very large center-of-mass energy, single-top and nontop production increase rapidly. The three processes become comparable in size for $e^{+} e^{-}$colliders operating in the multi$\mathrm{TeV}$ regime.

\section{Summary and conclusions}

The prospects for precision top quark physics at future lepton colliders relies on a comparison of measured cross-sections to very precise predictions of top quark pair production, including NLO and sometimes NNLO calculations. However, as we have shown in this note, single top events, that are ignored in most prospect studies, may lead to significant effects. A precise experimental separation of single top and top quark pair production processes seems difficult. For this reason we advocate the analysis of $W^{+} b W^{-} \bar{b}$ production, that includes single top quark production, and leads to increased statistics and possibly smaller systematic errors. To achieve the ultimate possible precision at the ILC, observables for the $W^{+} b W^{-} \bar{b}$ final state must be calculated with a precision well below $1 \%$, at NNLO, both at production threshold and in the continuum.

Acknowledgments The authors thank the WHIZARD authors, in particular Juergen Reuter and Fabian Bach, for their continued support. We thank Pedro Ruiz-Femenia for helpful discussions.

Open Access This article is distributed under the terms of the Creative Commons Attribution 4.0 International License (http://creativecomm ons.org/licenses/by/4.0/), which permits unrestricted use, distribution, and reproduction in any medium, provided you give appropriate credit to the original author(s) and the source, provide a link to the Creative Commons license, and indicate if changes were made.

Funded by SCOAP S $^{3}$

\section{References}

1. The ILC Community, The international linear collider technical design report-volume 1: executive summary. arXiv:1306.6327

2. The ILC Community, ILC reference design report: ILC global design effort and world wide study. arXiv:0712.1950

3. M. Aicheler, M. Aicheler, P. Burrows, M. Draper, T. Garvey et al., A multi-TeV linear collider based on CLIC technology, CERN2012-007

4. H. Baer, T. Barklow, K. Fujii, Y. Gao, A. Hoang et al., The international linear collider technical design report-volume 2: physics. arXiv: 1306.6352

5. J.E. Brau, R.M. Godbole, F.R.L. Diberder, M. Thomson, H. Weerts et al., The physics case for an e+e- linear collider. arXiv: 1210.0202

6. The ILC Community, ILC reference design report volume 2: physics at the ILC. arXiv:0709.1893

7. American Linear Collider Working Group, T. Abe et al., Linear collider physics resource book for Snowmass 2001. 1: Introduction. arXiv:hep-ex/0106055, arXiv:hep-ex/0106056, arXiv:hep-ex/0106057, arXiv:hep-ex/0106058

8. American Linear Collider Working Group, T. Abe et al., Linear collider physics resource book for Snowmass 2001. 2: Higgs and supersymmetry studies. arXiv:hep-ex/0106056

9. American Linear Collider Working Group, T. Abe et al., Linear collider physics resource book for Snowmass 2001. 3: Studies of exotic and standard model physics. arXiv:hep-ex/0106057

10. American Linear Collider Working Group Collaboration, T. Abe et al., Linear collider physics resource book for Snowmass 2001. 4: Theoretical, accelerator, and experimental options. arXiv:hep-ex/0106058

11. ECFA/DESY LC Physics Working Group, J.A. Aguilar-Saavedra et al., TESLA technical design report part III: physics at an e+elinear collider. arXiv:hep-ph/0106315

12. L. Linssen, A. Miyamoto, M. Stanitzki, H. Weerts, Physics and detectors at CLIC: CLIC conceptual design report. arXiv: 1202.5940

13. P. Lebrun, L. Linssen, A. Lucaci-Timoce, D. Schulte, F. Simon et al., The CLIC programme: towards a staged $e^{+} e^{-}$linear collider exploring the Terascale: CLIC conceptual design report. arXiv: 1209.2543

14. CLIC Physics Working Group, Physics at the CLIC multi-TeV linear collider. arXiv:hep-ph/0412251

15. D. Asner, A. Hoang, Y. Kiyo, R. Pschl, Y. Sumino et al., Top quark precision physics at the International Linear Collider. arXiv: 1307.8265

16. K. Seidel, F. Simon, M. Tesar, S. Poss, Top quark mass measurements at and above threshold at CLIC. Eur. Phys. J. C 73, 2530 (2013). arXiv:1303.3758 
17. T. Horiguchi, A. Ishikawa, T. Suehara, K. Fujii, Y. Sumino et al., Study of top quark pair production near threshold at the ILC. arXiv: 1310.0563

18. M. Amjad, M. Boronat, T. Frisson, I. Garcia, R. Poschl et al., A precise determination of top quark electro-weak couplings at the ILC operating at $\sqrt{s}=500 \mathrm{GeV}$. arXiv:1307.8102

19. E. Boos, M. Dubinin, A. Pukhov, M. Sachwitz, H. Schreiber, Single top production in e+ e-, e- e-, gamma e and gamma gamma collisions. Eur. Phys. J. C 21, 81-91 (2001). arXiv:hep-ph/0104279

20. P. Batra, T.M. Tait, Measuring the W-t-b interaction at the ILC. Phys. Rev. D 74, 054021 (2006).arXiv:hep-ph/0606068

21. W. Kilian, F. Bach, T. Ohl, J. Reuter, WHIZARD 2.2 for linear colliders. arXiv: 1403.7433

22. W. Kilian, T. Ohl, J. Reuter, WHIZARD: simulating multi-particle processes at LHC and ILC. Eur. Phys. J. C 71, 1742 (2011). arXiv:0708.4233

23. ILD Concept Group Collaboration, T. Abe et al., The international large detector: letter of intent. arXiv:1006.3396

24. T. Behnke, J.E. Brau, P.N. Burrows, J. Fuster, M. Peskin et al., The international linear collider technical design report-volume 4: detectors. arXiv:1306.6329
25. GEANT4 Collaboration, S. Agostinelli et al., GEANT4: a simulation toolkit. Nucl. Instrum. Methods A 506, 250-303 (2003)

26. J.H. Kuhn, Weak interactions of quarkonia. Acta Phys. Polon. B 12, 347 (1981)

27. K. Fujii, T. Matsui, Y. Sumino, Physics at t anti-t threshold in e+ e- collisions. Phys. Rev. D 50, 4341-4362 (1994)

28. M. Martinez, R. Miquel, Multiparameter fits to the $t$ anti-t threshold observables at a future e+ e- linear collider. Eur. Phys. J. C 27, 49-55 (2003). arXiv:hep-ph/0207315

29. P. Marquard, A.V. Smirnov, V.A. Smirnov, M. Steinhauser, Quark mass relations to four-loop order. arXiv:1502.0103

30. A.H. Hoang, C.J. Reisser, P. Ruiz-Femenia, Phase space matching and finite lifetime effects for top-pair production close to threshold. Phys. Rev. D 82, 014005 (2010). arXiv:1002.3223

31. M. Beneke, B. Jantzen, P. Ruiz-Femenia, Electroweak nonresonant NLO corrections to $\mathrm{e}+\mathrm{e}-\rightarrow \mathrm{W}+\mathrm{W}-\mathrm{b}$ bbar in the t tbar resonance region. Nucl. Phys. B 840, 186-213 (2010). arXiv: 1004.2188 